\title{
Phase Offset Analysis of Asymmetric Communications Infrastructure in Smart Grid
}

\author{
Mohammad Kamrul Hasan ${ }^{1}$, Siti Hajar Yousoff ${ }^{2}$, Musse Mohamud Ahmed ${ }^{1}$, \\ Aisha Hassan Abdalla Hashim², Ahmad Fadzil Ismail ${ }^{2}$, Shayla Islam ${ }^{3}$ \\ ${ }^{I}$ Department of Electrical and Electronics Engineering, Universiti Malaysia Sarawak, \\ 94300 Kota Samarahan, Malaysia \\ ${ }^{2}$ Department of Electrical and Computer Engineering, International Islamic University Malaysia, \\ Kuala Lumpur, Malaysia \\ ${ }^{3}$ Department of Computer Science and Engineering, Green University of Bangladesh \\ hmkamrul@unimas.my
}

\begin{abstract}
Synchrophasor advancement has the conversant to the smart grid applications, which is turned into necessary integral parts of digital communication frameworks that include transmission of electrical signals measured crosswise over various parts, which synchronized the grid applications utilizing external precise time source. The existing Communication protocol IEC 61850 for substation automation in Smart Grid today is being used with the support of up to 12 RS232/485 and 6 Ethernet ports, SCADA server, and PMUs. However, there is still having timing and control issue in the digital smart grid applications. Therefore, this paper proposes the hybrid synchronization scheme. The design considerations of the proposed scheme are mainly IP enabled broadband connectivity, IEEE 1588 clock server, and C37.118 functionalities. The result shows that the proposed scheme performs better than the existing systems.
\end{abstract}

Index Terms-WAMS; PMU; Phase offset; Synchronization; Smart grid.

\section{INTRODUCTION}

Currently, the operation of smart grids is monitored and controlled using the supervisory control and data acquisition (SCADA), and Wide Area Measurement (WAM) system. Both SCADA and WAM systems are linked through communication infrastructure. The communication infrastructure can be wired/wireless local area network, RF, and microwave, as well as optical fiber to reassure grid system operation. They connect transmission substations with the main generators to facilitate an integrated system. The actual operation of communication systems in power grids occurs using lines along the system with advanced optical networks. Loss of these communication wires is possible and could make protection and monitor the network more complex. Using advanced wireless communication and sensing devices could improve the controlling and monitoring of the whole system. The advancement of PMU based WAM system has taken place of SCADA since SCADA provides updates of the variables in times that can

Manuscript received 27 May, 2018; accepted 6 October, 2018.

A special thanks to Research Management and Innovation Center, Universiti Malaysia Sarawak (UNiMAS) for the research grant F02/DPD/1639/2018. This work is also partially supported by the FRGS17038-0604, International Islamic University, Malaysia. reach up to 15 seconds which creates the complexity of the electric power system [1].PMUs and intelligent electronic devices are used in Wide Area Measurement (WAM) to measure, monitor and control the smart grid (Fig. 1) [2]. These devices are interconnected and can be communicated to the main IED, which is implemented in the substation. Moreover, PMUs and IEDs can monitor and update the electric flow status in real-time and can be used to manage and control the grid including the points of $\mathrm{HV}$ and $\mathrm{LV}$ exchange. However, the existing SCADA and PMU based WAM systems face the control, protection, timing, and security issues in the smart grid. The existing monitoring system that monitors through SCADA, provides updates on the variables at times that can reach up to 15 seconds depending on the complexity of the electric power system. However, the 15 second is too large in terms of synchronization in order to have the precise control signal, fault monitoring for the protection, timing issues in smart grid, and also the measurement of the variables is not precise since it produces the information with different time bases.

Most of these problems occur due to no synchronization and mal-operation of the protection system which relies on distance protection relays and traditional backup schemes. In this study, the advanced features of PMU will be utilized which has more efficiency in capturing the quick occurring disturbances or the resonance effects, as it uses GPS time synchronization. The synchronization problem is based on network sampling which has the following effects:

- Network time delay is uncertain

- External GPS clock is used for synchronization

- Strong dependence on time synchronization network

- External clock failure will bring sampling out of step, then relay function will be influenced and relay may not operate due to clock offset because of GPS lost. This problem can represent in Fig. 2.

Due to the lack of synchronization between the load zones and the control center, the faults occurred. So, it creates the issues to detect real-time faults at the protection system. One of the key parameters in synchronization is the delay. The communication link for smart grid can be designed using 
wireless/wired fiber optics medium to connect the PMUs, in which case the receive delays, propagation delays may raise. Moreover, the various influencing factors such as delays of the transducer, a window size of the DFT, processing time, and the large volume of the data at PMU output viand the other communication associates delays [3]. Therefore, these delays are needed to estimate in WAMS of the smart grid. The communication delays can be stated in (1) [3], [4].

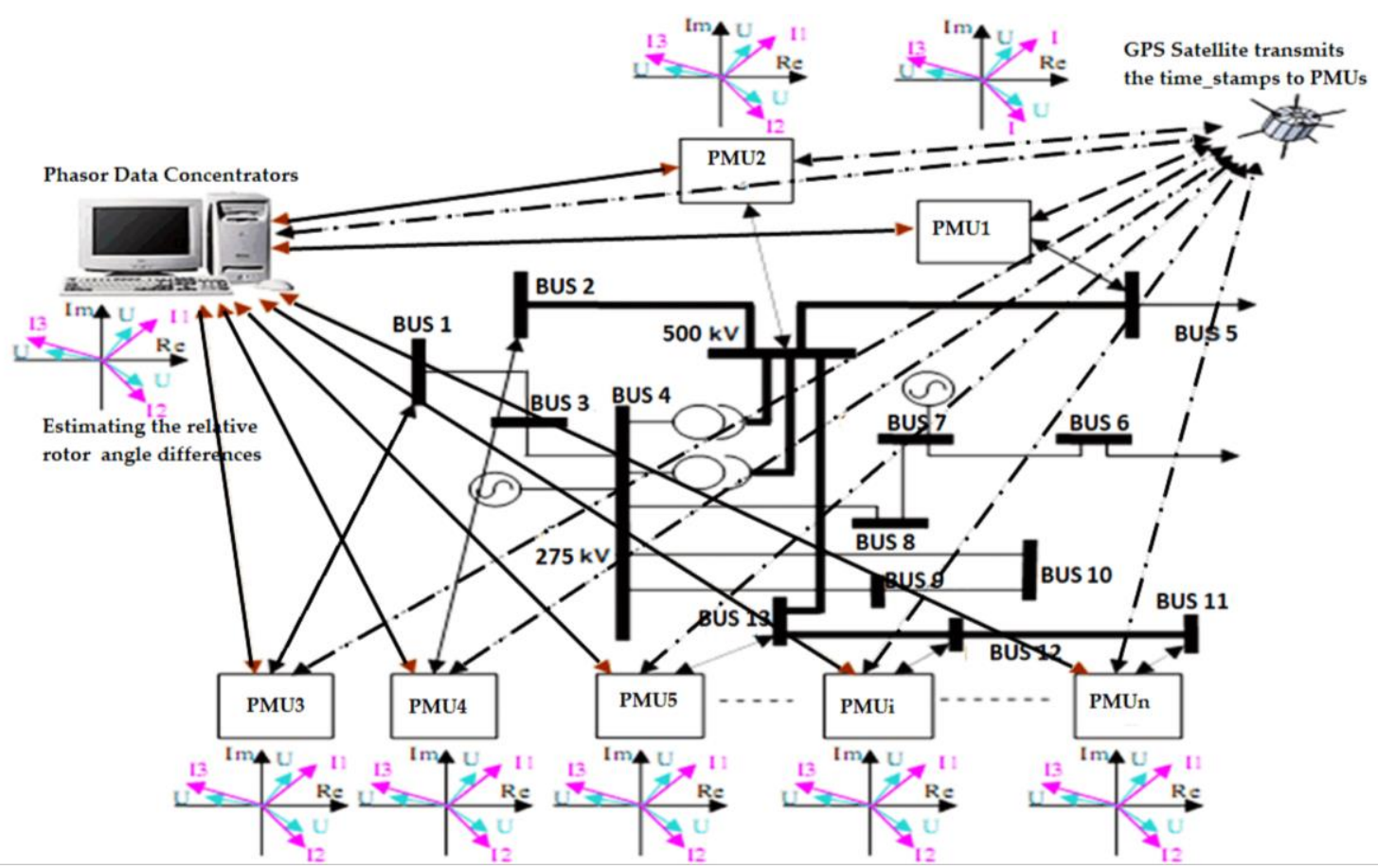

Fig. 1. GPS enabled Communication Framework for WAM System in Smart Grid Applications [2].

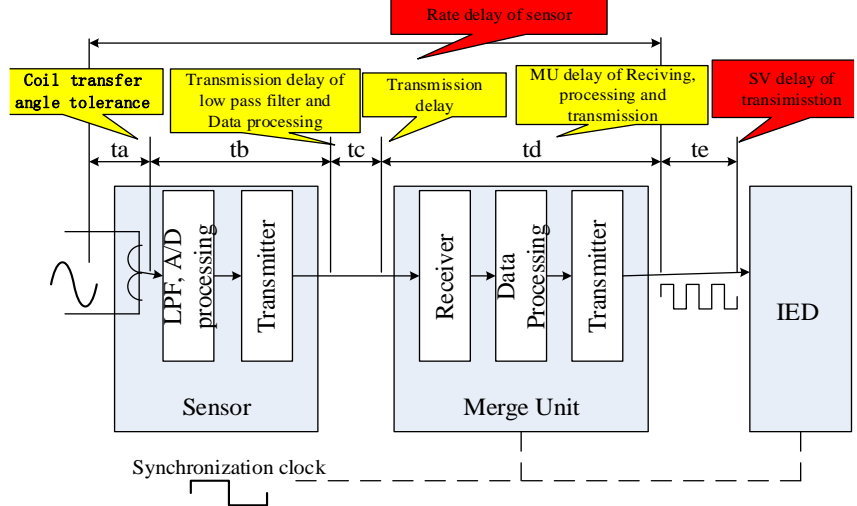

Fig. 2. Clocks problem for time synchronization.

$$
\tau=\tau_{f}+\tau_{p}+\frac{L}{R}+\phi
$$

where $\tau$ is the total link delay, $\tau_{f}$ states the fixed delay allied with transducers, DFT processing, data concentration, and multiplexing, $\tau_{p}$ presents the link propagation delay, $L$ is the transferred data, $R$ states data rate of the communication link, and network arbitrary jitter can be itemized by $\phi$.

The trend of measurement of the synchronized real-time positive structure of voltage magnitude and current phase angle of a power system has obtained by the latest PMUs in a WAM system. The synchronization process normally can be achieved through the usage of the exact sample of voltage and current waveforms. This is completed through the timing signals of a global time timestamps. The current trend of this usage of the reference frame is from the GPS Satellite (Fig. 3) [3], [5].

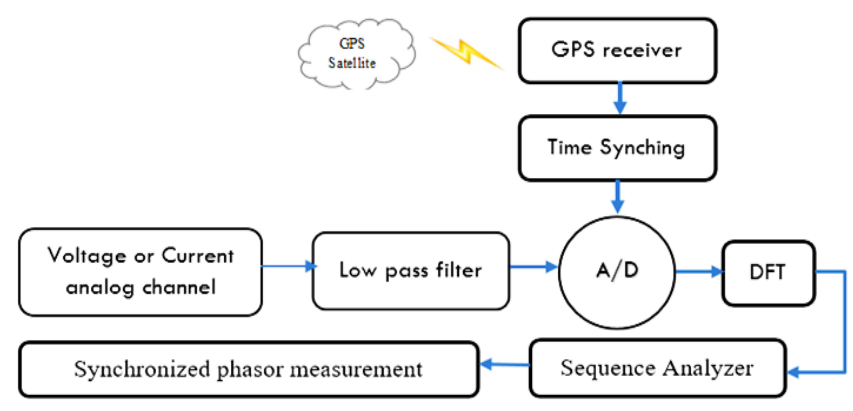

Fig. 3. Synchronized phasor measurement structure.

At the smart grid, the analog power signal is normally transformed into pieces of digital data by using the analog to digital (A/D) converter. For instance, for the voltage .measurement phase samples are taken for each cycle of the waveform and then using DFT the fundamental frequency component is estimated.

Using the PMU, synchronized phasor signals can be signified in (2) [6]

$$
\begin{gathered}
x(t)=x r+j x i=\left(\frac{x m}{\sqrt{2}} e^{\varphi}\right)= \\
=\frac{x m}{\sqrt{2}}(\cos \varphi+j \sin \varphi),
\end{gathered}
$$

where $x m$ states the amplitude of synchronized vector, phase angle is denoted by $\varnothing$ and $x i$ and $x r$ are imaginary and real components of complex value. The general method for phasors calculation by using sample data is discrete Fourier transform. Phasors of the three stages are consolidated to create the positive arrangement estimation. The figure incorporates an equipment low-pass filter (Hardware LPF) 
for anti-aliasing and a simple to-computerized (A/D) converter for simple to-advanced change.

PMUs measure the transferred voltage(s) and all the significant line streams. These estimations are sent to a Phasor Data Concentrator (PDC) at the control focus. The PDC corresponds the information by time tag to make a framework wide estimation [7]. The PDC sends out these estimations as an information stream when they have been gotten and related. Framework security focus (SPC) get Datastream and make a wide region assurance relying upon the wide-zone see. This guideline of activity will be utilized as a part of this examination.

The arrangement of supervision allows storing the records of a similar occasion at various focuses in the power system with a GPS based time reference. The phasor estimating unit is spoken to by a discrete stage succession analyser piece which changes over 3 stage signals (Vabc or Iabc) to a positive, negative and zeroes grouping part sizes and edge. Each stage flag ( $\mathrm{Va}, \mathrm{Vb}$, and $\mathrm{Vc}$ ) is changed over to an accurate positive and exact sequence that utilizing by the DFT. The positive sequence component can be shown in (3) [3]

$$
V_{1}=\frac{1}{3}\left(V_{a}+\alpha \times V_{b}+\alpha^{2} \times V_{c}\right)
$$

where $\alpha=j \angle 120^{\circ}$. IEEE C37.118 is the enhanced for synchrophasor correspondence framework standard that communicates the smart grid applications [8]. It defines strategies for assessment of synchrophasor estimations, time synchronization, use of time-stamps and format of messages traded over the system. It doesn't put any confinement on the communication mode, protocol or algorithm and messages can be transmitted in unicast, multicast or communicate form over any correspondence medium and transport convention. Initially, IEEE C37.118 tended to the execution of synchrophasors just under consistent state conditions disregarding framework unsettling influences and commotion [9], [10].

The main aim of this study is to assess the phase offsets for the asymmetric communication infrastructure and also to reduce the phase offset errors in smart grid applications. Contributions of this study are listed as follows:

1. A hybrid synchronization scheme is proposed to improved phase offset errors in smart grid applications.

2. The performance of the proposed scheme is assessed through simulation approach. The considered performance metric is the phase offset error.

\section{PROPOSED SYNCHRONIZATION SCHEME}

This research mainly focuses on the phasor measurement units and their synchronizations to have the precise communications of measuring the real-time data from the smart grid-enabled digital substations. Smart Grid Synchronization to an external timescale is typically provided by the GPS, which allows receivers to achieve an accuracy of some tenth of nanoseconds. GPS collectors are too vast and exorbitant, and furthermore, require excessive energy to be incorporated on a little sensor hub. The NTP can disperse to organize PCs the time which is assessed by methods for GPS [11]. Gives a period reference and, in fixed organize applications, it can circulate synchronization among arranged PCs. To facilitates, the season of a sender to all recipients a period stamp has utilized [12]-[15]. Reference communicate messages don't contain an unequivocal timestamp; recipients utilize the communicate message landing time to alter their timekeepers. The messages are communicated at the physical layer and after that they touch base at the collectors' changeability. In [16], a calculation is suggested that with a specific end goal to synchronize the whole system needs a way that contains all sensor hubs. The primary thought is to communicate something specific alongside a circle recording bounce by jump the underlying time and the end time of the message. A roundtrip transmission with the immediate neighbours is performed to assess the normal transmission delay and the standard deviation. Typically, storing timing records from the others components is to adjust the local time with an accurate estimation of the delay with a minimum delay. After a rigorous assessment of the existing systems challenges, it is necessary to overcome the synchronization issues in order to solve the control, protection, timing, and security issues in the smart grid. The IEEE C37.118 communication protocol is needed to enhance in terms of security, and timing as well as accuracy. The proposed model considers the PMU, PDC units, private tunnelled WLAN, and mobile network for the real-time timestamps. The following architectures and block diagrams are also considered in developing the real-time synchronization scheme for the PMU based smart grid applications..

A synchronization scheme is proposed with the extension of IEEE C37.118 to synchronize the asymmetric PMUs. In the proposed scheme the phase offset estimation is discussed below:

At first, the external synchronization reference such as IEEE1588 clock server transmitted the sync timestamp message using broadband connectivity in order to transmit to this beacon message to PMUs in a smart grid system. The timing diagram is presented in Fig. 4.

Then, $i^{\text {th }}$ PMUs receives this beacon message and request for synchronized timestamps. At the time it receives the timestamps PMUS saves the influx time $\left(T_{n}^{P M U_{A \rightarrow C}}\right)$ compare self-clocks. Thereby, $i^{\text {th }}$ PMUs will exchange clocks as well as updates the Sync_time. Finally, it sends an acknowledgment to the PDC and PMUs also sends the acknowledgment (AcK) to the PDCs. By then it can estimate the delay difference for both of send and receive, propagation delay to figure out the phase offset errors. Considering delay difference for both of send and receive, propagation delay, and frequency errors the phase offset can be estimated by the following

$$
\begin{aligned}
T_{A \rightarrow C}^{\text {Sync }}= & \Phi_{A \rightarrow C}^{f_{\text {error }}}\left\{\begin{array}{l}
T_{1}^{P M U_{A \rightarrow C}}+\frac{\alpha}{2}+ \\
\frac{P M U_{A \rightarrow C} \rho_{\text {uplink }}^{P M U_{C}}}{\rho_{\text {uplink }}}
\end{array}\right\}+ \\
& +\widehat{T}_{\text {offset }}^{P M U_{A \leftarrow C}}+\frac{\vartheta_{C}^{\text {delay }}-\vartheta_{A}^{\text {delay }}}{2},
\end{aligned}
$$




$$
\begin{aligned}
& \widehat{T}_{\text {offset }}^{\text {PMU }} A \leftarrow C \cong \frac{1}{M} \sum_{i=1}^{M} \widehat{T}_{\text {offset }}^{P M U_{A}} A \leftarrow C, \\
& \rho_{\text {uplink }}^{P M U_{A} \rightarrow C}=\frac{\rho_{\text {uplink }}^{P M U_{C}}-\rho_{\text {uplink }}^{P M U_{A}}}{2},
\end{aligned}
$$

$$
\chi_{A \rightarrow C}^{\text {delay }}=\left[\begin{array}{l}
\left(\rho_{\text {total }}^{P M U_{A}}-\chi_{\text {rec }}^{P M U_{B}}\right)+\left(\chi_{\text {rec }}^{P M A_{B}}-\chi_{\text {rec }}^{P M U} C\right. \\
\left(\chi_{\text {rec }}^{P M U_{C}}-\chi_{\text {rec }}^{P M U_{A}}\right)
\end{array}\right] .
$$

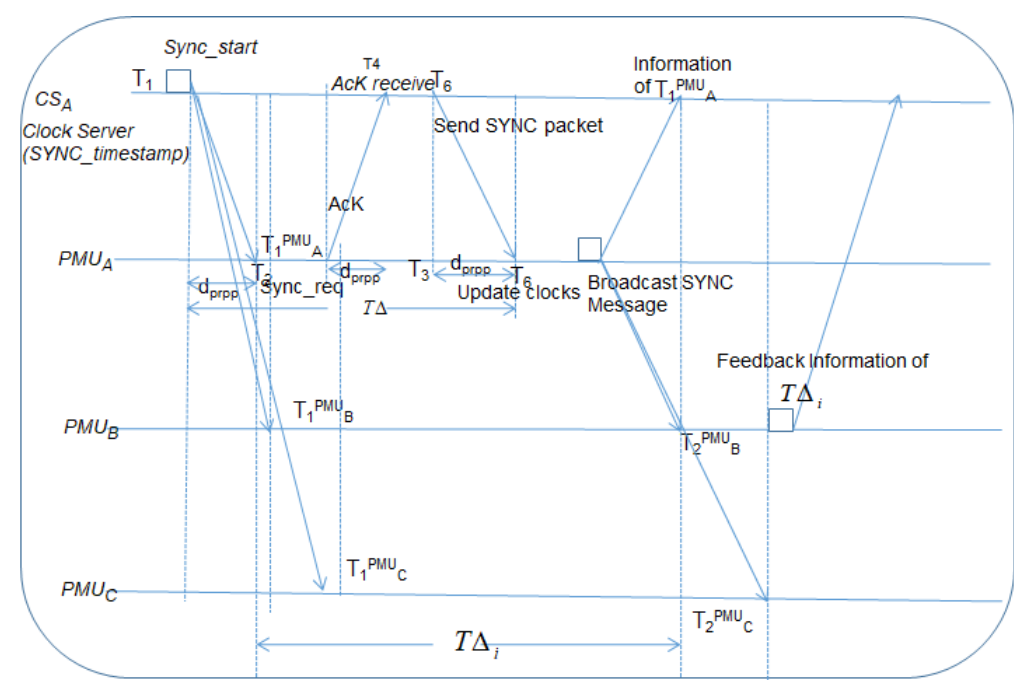

Fig. 4. A timing diagram for the hybrid synchronization scheme.

In the proposed scheme a synchronization signal contains the synchronization message packets (Sync_message) level that is broadcasted from the master clock server generated contains the header ID, Sync_clk, number of hops and Synchronization Status Message Byte. The system is then automatically organized in such a way that, when one PMU receives more than one synchronization signals from the master clock server then the PMUs estimates and adjusts the clocks between with and also sends the feedback to the main server. The feedback message contains the delay time, propagation information and status of the synchronization.

\section{RESULTS AND DISCUSSION}

The performance of the proposed scheme is evaluated using Monte Carlo simulation. It is recorded that if there is asymmetry speed occurred in communication links there a lot of errors are also introduced in the synchronization system. As a result of the complete systems phase offsets errors are enhanced and system outages reached higher. So, the asymmetric link speed and transmission rate cannot be fixed, especially during low bandwidth connectivity in smart grid infrastructure. The simulation parameters are listed in Table I.

TABLE I. SIMULATION PARAMETERS [1], [14]-[16].

\begin{tabular}{|c|c|}
\hline Factors & Values \\
\hline PDCs & 6 \\
\hline PMUs & 30 \\
\hline Delay variation & $1 \mathrm{~ms}, 10 \mathrm{~ms}, 100 \mathrm{~ms}$ \\
\hline Synchronization Trials & 10 \\
\hline Number of samples for Monte Carlo & 1024 \\
\hline Carrier bandwidth & $10 \mathrm{MHz}$ \\
\hline Time-bandwidth factor & $100 \mathrm{MHz}$ \\
\hline Bandwidth & $10 \mathrm{Mbps}$ \\
\hline Connectivity & IP Connectivity \\
\hline
\end{tabular}

Figure 4, phase offset error for the proposed scheme is evaluated considering the communication link speed variations. These variations have some correlation with receive delay deviations $(1 \mathrm{~ms} / 10 \mathrm{~ms} / 100 \mathrm{~ms})$. So, the Fig. 5 shows the phase offset errors with this speed and delay variations.

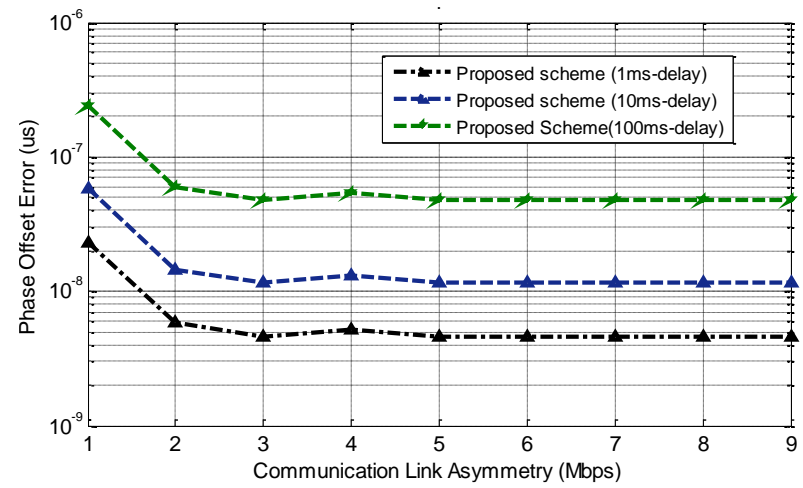

Fig. 5. Phase offset error vs. Communication link asymmetry. The recorded various connectivity speed such as $1 \mathrm{Mbps}$ to $9 \mathrm{Mbps}$, with the delay of $1 \mathrm{~ms}, 10 \mathrm{~ms}$, and $100 \mathrm{~ms}$.

Figure 6 demonstrated the phase offset errors estimations for the proposed and the existing scheme. The point should be noted that the IP communication connectivity was considered for the proposed scheme.

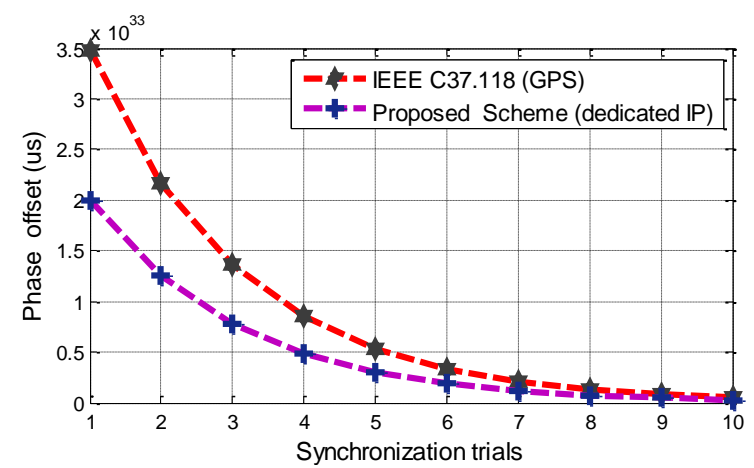

Fig. 6. Phase offsets vs. synchronization trials while the receive delay deviation was considered to $100 \mathrm{~ms}$.

Figure 6 also demonstrated that the initial phase offset is 
higher. However, applying several trials with Sync_time samples the offset errors can reduce. It is evident from the plot that the proposed scheme is better than that of an existing algorithm.

\section{CONCLUSIONS}

This paper investigates the key synchronization for smart grid communications infrastructure. Typically, the existing smart grid synchronization is designed using external timestamps that are typically provided by the GPS satellite. However, it has been assessed that the GPS enabled smart grid synchronization is troublesome while the GPS is unable to locate by the PMU receivers. As a result of asynchronous and error occurs in measuring the real-time data. Moreover, the study also suggests that the existing system still has the lack of standard data names prevent auto-discovery and selfdescription without knowledge of configuration messages. Therefore, this paper is proposed the hybrid synchronization scheme. The hybrid synchronization is designed by the consideration of IP connectivity, IEEE 1588. The performance of the proposed scheme performs better than the existing system. The future recommendation of this study can be an enhanced protocol with the hybrid functionalities of IEEE 1588 and C37.118.

\section{REFERENCES}

[1] R. M. Moraes, H. A. R. Volskis, Yi Hu, "Deploying a large-scale PMU system for the Brazilian interconnected power system", in Third Int. Conf. Electric Utility Deregulation and Restructuring and Power Technologies, 2008, pp. 143-149. DOI: 10.1109/DRPT.2008.4523392.

[2] M. Alexandru et al., "From SCADA to smart grid in power transmission and distribution systems", Bulletinul, General Association of Engineers in Romania (AGER), 2011.

[3] M. M. Eissa, M. E. Masoud, M. M. M. Elanwar, "A novel back up wide area protection technique for power transmission grids using phasor measurement unit", IEEE Trans. Power Deliv., vol. 25, no. 1, pp. 270-278, 2010. DOI: 10.1109/TPWRD.2009.2035394.

[4] R. Klump, R. E. Wilson, K. E. Martin, "Visualizing real-time security threats using hybrid SCADA/PMU measurement displays", in Proc. 38th Annual Hawaii Int. Conf. System Sciences, 2005, pp. 55c-55c. DOI: 10.1109/HICSS.2005.679.
[5] L. A. Kumar, S. Karthikeyan, "Modeling of phasor measurement unit for wide area monitoring and control of smart grids with distributed energy resources", in IEEE Conf. Technologies for Sustainability (SusTech 2016), 2016, pp. 188-194. DOI: 10.1109/SusTech.2016.7897165.

[6] S. N. Muneshwar, R. P. Hasabet, P. Kose, A. A. Bhole, "A new adaptive PMU based protection scheme for interconnected transmission network system", in 2014 Int. Conf. Circuits, Power and Computing Technologies (ICCPCT 2014), 2014, pp. 179-183. DOI: 10.1109/ICCPCT.2014.7054922.

[7] G. Renal, M. Kanabar, V. Muthukrishnan, "Practical aspects of testing Phasor Data Concentrators for wide area monitoring systems", in IEEE 27th Canadian Conf. Electrical and Computer Engineering (CCECE 2014), 2014, pp. 1-6. DOI: 10.1109/CCECE.2014.6901061.

[8] K. E. Martin, D. Hamai, M. G. Adamiak, S. Anderson, M. Begovic, G. Benmouyal, G. Brunello, "Exploring the IEEE standard C37. 1182005 synchrophasors for power systems", IEEE Trans. Power Delivery, vol. 23, no. 4, 2008, pp. 1805-1811. DOI: 10.1109/TPWRD.2007.916092.

[9] D. Fan, V. Centeno, "Phasor-based synchronized frequency measurement in power systems", IEEE Trans. Power Deliv., vol. 22, no. 4, pp. 2010-2016, 2007. DOI: 10.1109/TPWRD.2007.905371

[10] E. O. Schweitzer, D. Whitehead, G. Zweigle, K. G. Ravikumar, "Synchrophasor-based power system protection and control applications", in 63rd Annual Conf. Protective Relay Engineers, 2010, pp. 1-10. DOI: 10.1109/CPRE.2010.5469481.

[11] V. Shankarkumar, L. Montini, T. Frost, G. Dowd, "Precision Time Protocol Version 2 (PTPv2) Management Information Base", Jun. 2017. DOI: $10.17487 /$ RFC8173.

[12] M. Maroti, B. Kusy, G. Simon, A. Ledeczi, "The flooding time synchronization protocol", in Proc. 2nd Int. Conf. Embedded Networked Sensor Systems (SenSys 2004), 2004, p. 39. DOI: 10.1145/1031495.1031501.

[13] M. K. Hasan, R. A. Saeed, R. A. Alsaqour, A. F. Ismail, H. A. Aisha, S. Islam, "Cluster-based time synchronisation scheme for femtocell network", International Journal of Mobile Communications, vol. 13, no. 6, p. 567, 2015. DOI: 10.1504/IJMC.2015.072063.

[14] M. K. Hasan, A. F. Ismail, W. Hashim, S. Islam, M. H. Khairolanuar, "Effects of phase offset error for asymmetric communication link in heterogeneous mobile network", Lecture Notes in Electrical Engineering, pp. 957-966, 2016. DOI: 10.1007/978-3-319-245843_81.

[15] M. K. Hasan et al., "A self-organizing approach: time synchronization for the HeNodeBs in heterogeneous network", Lecture Notes in Electrical Engineering, pp. 365-374, 2016. DOI: 10.1007/978-3-31924584-3_30.

[16] K. S. Yildirim, R. Carli, L. Schenato, "Adaptive proportional-integral clock synchronization in wireless sensor networks", IEEE Trans. Control Systems Technology, vol. 26, no. 2, pp. 610-623, 2018. DOI: 10.1109/TCST.2017.2692720. 